\title{
BMJ Open Associations of coprescribed medications for chronic comorbid conditions in very old adults with clinical dementia: a retrospective cohort study using insurance claims data
}

\author{
Nobuhiro Handa (10 , ${ }^{1,2,3,4}$ Seigo Mitsutake, ${ }^{1}$ Tatsuro Ishizaki, ${ }^{1}$ Tetsuo Nakabayashi, ${ }^{3}$ \\ Masahiro Akishita, ${ }^{5}$ Nanako Tamiya, ${ }^{6}$ Satoru Yoshie, ${ }^{7}$ Katsuya lijima ${ }^{7,8}$
}

To cite: Handa N, Mitsutake S, Ishizaki T, et al. Associations of coprescribed medications for chronic comorbid conditions in very old adults with clinical dementia: a retrospective cohort study using insurance claims data. BMJ Open 2021;11:e043768. doi:10.1136/ bmjopen-2020-043768

- Prepublication history and additional supplemental material for this paper are available online. To view these files, please visit the journal online (http://dx.doi.org/10.1136/ bmjopen-2020-043768)

Received 13 August 2020 Accepted 08 June 2021

Check for updates

(C) Author(s) (or their employer(s)) 2021. Re-use permitted under CC BY-NC. No commercial re-use. See rights and permissions. Published by BMJ.

For numbered affiliations see end of article.

Correspondence to Dr Nobuhiro Handa; handa-nobuhiro@pmda.go.jp

\section{ABSTRACT}

Objective To assess the association of coprescribed medications for chronic comorbid conditions with clinical dementia in older adults, as indicated by the initiation of a new prescription of antidementia medication (NPADM). Design Retrospective enumeration cohort study. Setting A Japanese city in Tokyo Metropolitan Area. Participants A total of 42024 adults aged $\geq 77$ years residing in Kashiwa City, a suburban city of Tokyo Metropolitan Area, who did not have any prscription of antidementia medication from 1 April to 30 June 2012. Main outcome measure The primary outcome was NPADM during follow-up period until 31 March 2015 (35 months). Subjects were categorised into four age groups: group 1 (77-81 years), group 2 (82-86 years), group 3 (87-91 years) and group 4 ( $\geq 92$ years). In addition to age and sex, 14 sets of medications prescribed during the initial background period (from 1 April 2012 and 31 June 2012) were used as covariates in a Cox proportional hazard model.

Results In a follow-up period of 1345457 personmonths (mean $=32.0 \pm 7.5$ months, median 35 months), NPADM occurred in 2365 subjects. NPADM incidence at 12 months was $1.9 \% \pm 0.1 \%$ (group $1: 0.9 \% \pm 0.1 \%$, group $2: 2.1 \% \pm 0.1 \%$, group $3: 3.2 \% \pm 0.2 \%$ and group $4: 3.6 \% \pm 0.3 \% ; p<0.0001)$. In addition to older age and female sex, use of the following medications was significantly associated with NPADM: statins (HR: 0.82 , $95 \% \mathrm{Cl} 0.73$ to $0.92 ; p=0.001$ ), antihypertensives (HR: $0.80,95 \% \mathrm{Cl} 0.71$ to $0.85 ; p<0.0001)$, non-steroidal bronchodilators (HR: $0.72,95 \% \mathrm{Cl} 0.58$ to $0.88 ; \mathrm{p}=0.002$ ), antidepressants (HR: $1.79,95 \% \mathrm{Cl} 1.47$ to 2.18; $\mathrm{p}<0.0001$ ), poststroke medications (HR: $1.45,95 \% \mathrm{Cl} 1.16$ to 1.82; $p=0.002$ ), insulin (HR: $1.34,95 \% \mathrm{Cl} 1.01$ to 1.78 ; $\mathrm{p}=0.046$ ) and antineoplastics (HR: $1.12,95 \% \mathrm{Cl} 1.01$ to $1.24 ; p=0.035$ ).

Conclusions This retrospective cohort study identified the associations of coprescribed medications for chronic comorbid conditions with NPADM in older adults. These findings would be helpful in understanding the current clinical practice for dementia in real-world setting and potentially contribute to healthcare policymaking. Trial registration number UMIN000039040.

\section{Strengths and limitations of this study}

- This retrospective cohort study included all community-dwelling residents (aged $\geq 77$ years) in a Japanese city in April 2012 who were followed up for over 35 months ( $N=42024)$.

- A complete enumeration survey of national insurance record managed by a single payer, that is, the local government, was performed.

- The associations of multiple coprescribed medications for chronic comorbid conditions with onset of clinical dementia as indicated by initiation of a new prescription of antidementia medication were identified.

- Due to its retrospective observational nature, this study was not able to assess the cause-effect relationship of coprescribed medications in clinical dementia.

- Confounding factors such as lifestyle management and underlying disease were not evaluated.

\section{INTRODUCTION}

Dementia is a chronic neurodegenerative disorder that slowly progresses over the years. It is characterised by cognitive decline, memory loss and social functioning impairment and often culminates in severe disability and dependency among older patients, thereby imposing an immense burden on their families and caregivers. Dementia is a serious global health problem, with approximately 50 million cases worldwide in 2015 and nearly 10 million new diagnoses annually, and at this rate expected to triple by $2050 .{ }^{1}$

Current antidementia medications can only alleviate symptoms, and there are no definitive treatment modalities to prevent or reverse the disease. ${ }^{2}{ }^{3}$ Therefore, the management of dementia aims mainly to delay onset and slow its progression through 
attenuation of risk factors. In 2019, the WHO issued guidelines to reduce the risk of cognitive decline and dementia, including recommendations for increased physical activity, increased social engagement, tobacco cessation, alcohol use reduction, nutritional management, weight management, cardiometabolic risk factor (hypertension, diabetes and dyslipidaemia) management and hearing loss management. ${ }^{1-3}$ However, the effects of these multimodal interventions are difficult to assess in real-world conditions due to the slow progression of cognitive decline and the need for multiple evaluations of patients over time. To understand the effects of such interventions, assessments must involve large numbers of participants, long-term observations and regular medical examinations with standardised cognitive tests and/or imaging tests.

An integral component of Japan's universal healthcare system is the Long Life Medical Care System (LLMCS) (online supplemental material 1), which provides insurance coverage to all Japanese residents aged 75 years or older and those aged 65-74 years with prespecified disabilities. ${ }^{4}$ Many of the older adults covered by the LLMCS take numerous medications for existing conditions, including known dementia risk factors. ${ }^{5}$ The associations between the pharmaceutical management of these conditions and the progression to clinical dementia, which requires pharmacological management, should be elucidated to manage and prevent disease onset.

In a complete enumeration of LLMCS data obtained from Kashiwa City, a suburban Japanese city in Tokyo Metropolitan Area, we examined if the use of prescription medications for existing chronic diseases is associated with subsequent initiation of a new prescription of antidementia medication (NPADM). This study focused on medications indicated only for Alzheimer's disease and Lewy body dementia with Japanese regulatory approval.

\section{METHODS}

This retrospective cohort study was conducted using insurance claims data of individuals residing in Kashiwa City, a typical satellite city with approximately 420000 residents located in the suburbs of the Tokyo Metropolitan Area. Under Japan's universal healthcare system, citizens and foreign nationals residing in Japan for 3 months or longer are required to enrol in an insurance programme. These include the LLMCS (for people aged $\geq 75$ years), national insurance programmes provided by local governments (for unemployed, self-employed and retired people aged $<75$ years), employees' insurance (for employed people aged $<75$ years and their dependents) and other various insurance programmes (online supplemental material 1$).{ }^{4}$

LLMCS data encompass complete claims records of residents, including outpatient records, hospitalisation records, prescription information, codes of medical supplies used during treatment and medical procedure codes. Thus, LLMCS data sets can be applied to analyse use of specific prescription medications in defined time periods.

We obtained Kashiwa City's LLMCS insurance claims data from April 2012 to March 2015. These records were linked with the city's Basic Resident Register, which contains records of deaths and movement of residents out of the city (which disqualifies an individual from the city's LLMCS). In the insurance claims data, age is categorised into 5-year intervals based on birth year (19211925, 1926-1930, 1931-1935, 1936-1940 and so forth). Although we sought to analyse residents aged 75 years or older (who qualify for LLMCS coverage), the 5-year age categorisation prevented the precise identification of these individuals. Therefore, we included all residents aged 77 years or older (born in 1935 or earlier) on 1 April 2012. Because not all residents aged 72-76 years (born in 1936-1940) would be enrolled in the LLMCS, this age group was excluded from analysis.

The Medical Practitioners' Act of Japan mandates that physicians can only prescribe a medication for a maximum of 3 months at a time. For this analysis, we set an 'initial background period' (IBP) of 3 months from 1 April to 30 June 2012 to identify each subject's baseline use of prescription medications for existing chronic diseases. From the 44302 eligible residents, those who had died $(n=365)$ or moved out of Kashiwa City $(n=129)$ during the IBP were further excluded from analysis and 18 more due to their residency in a nursing care facility on 1 April 2012, who were therefore covered by the long-term care insurance system (online supplemental material 1). We identified patients $(n=1766)$ who were already being treated with antidementia medication (cholinesterase inhibitors: donepezil, galantamine and rivastigmine; $\mathrm{N}$-methyl-d-aspartate receptor antagonist: memantine) for a separate analysis. Accordingly, the main analysis was conducted on 42024 residents who were not treated with any antidementia medication during the IBP (figure 1).

\section{Identification of prescription medications}

Each subject's prescribed medications for existing chronic disease were identified using insurance claims data. In order to be reimbursed under the LLMCS, healthcare providers submit claims to the Health Insurance Claims Review \& Reimbursement Services, which processes claims for pharmaceutical products approved for the Japanese market using specific billing codes. There were 200092 and 200597 billing codes for approved pharmaceutical products in 2012 and 2015, respectively. Medications for dementia risk factors were categorised into 15 groups based on their pharmacological action: antidementia medications (215 codes), antidiabetics (482), antihypertensives (1762), statins (333), antidepressants (349) and benzodiazepines (157), among others (online supplemental material 2). Poststroke medications that stimulate brain metabolism or increase cerebrovascular blood flow are also approved for use in patients who had stroke and were analysed. 
Kashiwa city residents aged $\geq 77$

years enrolled in the Long Life

Medical Care System as of Apr 1 , $2012(n=44,302)$

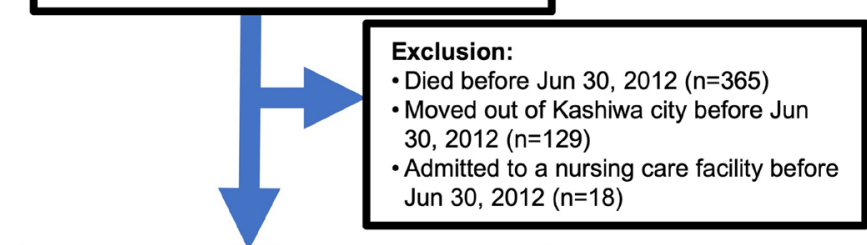

Residents with a complete profile of

prescribed medications during the initial

background period (Apr 1 to Jun 30, 2012)

$(n=43,790)$

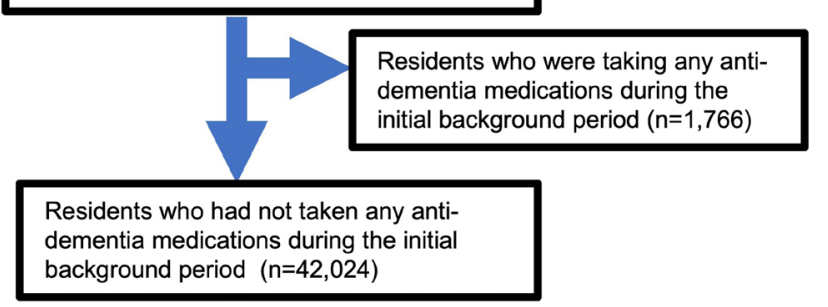

Figure 1 Study flow diagram. Initial background period: from 1 April 2012 to 31 June 2012.

\section{Outcome measure and statistical analysis}

The outcome measure was NPADM. The first antidementia medication, donepezil, was approved for managing the symptoms of Alzheimer's disease and Lewy body dementia in Japan by the Ministry of Health, Labour and Welfare in 1999. Since then, two more cholinesterase inhibitors (galantamine and rivastigmine) and one N-methyl-daspartate receptor antagonist (memantine) have been approved for the same indications. New prescriptions of any of these drugs were considered NPADM.

The observation period spanned a maximum of 35 months from April 2012 to March 2015. Subjects were censored if they died, moved out of Kashiwa City or were admitted to a nursing care facility. As the insurance coverage of individuals shifted from the LLMCS to longterm care insurance after admission to a nursing care facility, their LLMCS insurance claims data would be incomplete after this shift. Therefore, these subjects were censored in the month of their admission to a nursing care facility. All subjects were stratified into four age groups (group 1: age 77-81 years, group 2: age 82-86 years, group 3: age $87-91$ years and group 4: age $\geq 92$ years).

For the statistical analyses, continuous variables were calculated as mean values and SD, and differences were evaluated using unpaired t-test. Categorical variables were presented as frequency counts and proportions, and differences were evaluated using the likelihood ratio test. Cumulative incidence plots of NPADM for patient age, sex and prescribed medications were generated using the Kaplan-Meier method with log-rank test for comparisons. A Cox proportional hazards model was constructed for the multivariate analysis, with patient age, sex and 14 prescribed medications as covariates. Results were expressed as HR and 95\% CI. As the average life expectancy in the Japanese population is different between the two sexes (male, 80.8 years old; female, 87.0 years in 2015 ), sex difference may be associated with age. We performed ad hoc analyses to include an interaction term (age*sex) into the multivariable analysis. All statistical analyses were performed using SPSS V.25 software. Two-tailed $p$ values below 0.05 were considered significant.

\section{Patient and public involvement}

There was no patient involvement in this study.

\section{RESULTS}

\section{Coprescribed medications and incidence of NPADM}

This retrospective cohort study consisted of 42024 adults aged $\geq 77$ years who did not take any antidementia medication on 1 April 2012. They were followed up for over 35 months until 31 March 2015. The covariates were 14 groups of medications prescribed between 1 April and 31 June 2012 (IBP). More than 56\% of the 42024 study subjects were taking prescription medications from one target medication group during the IBP, 21.9\% from three groups and $4.1 \%$ from five groups. The total follow-up period was 1345457 person-months (mean \pm SD: $32.0 \pm 7.5$ months, median: 35 months), with 34212 $(81.4 \%)$ subjects completing the full follow-up period of 35 months. NPADM occurred in 2365 (5.6\%) subjects. A total of $5447(13.2 \%)$ subjects were censored due to death $(n=3899)$, moving out of Kashiwa City $(n=669)$ or admission to a nursing facility $(\mathrm{n}=879)$. The incidence of NPADM at 12 months was $1.9 \% \pm 0.1 \%$ (group 1: $0.9 \% \pm 0.1 \%$, group $2: 2.1 \% \pm 0.1 \%$, group $3: 3.2 \% \pm 0.2 \%$ and group 4: $3.6 \% \pm 0.3 \%$; $\mathrm{p}<0.0001$ ) (figure $2 \mathrm{~A}$ ). Women had a higher incidence of NPADM (figure 2B). The proportion of women and the prescribed medications during the IBP according to age group are presented in table 1. Approximately $56.6 \%$ of the subjects were women. During the IBP, $17.0 \%$ of subjects were taking statins, $41.0 \%$ were taking antihypertensives, $9.1 \%$ were taking antidiabetics (either insulin, oral hypoglycaemic agents or both), $2.5 \%$ were taking antidepressants and $2.2 \%$ were taking poststroke medications (table 1 ).

\section{Univariate and multivariate analyses}

The results of the Kaplan-Meier analysis (figures 2-5) showed that subjects who were taking the following medications during the IBP had significantly higher NPADM incidence: poststroke medications $(\mathrm{p}<0.0001)$, antidepressants $(\mathrm{p}<0.0001)$, antineoplastics $(\mathrm{p}<0.0001)$, antiplatelets $(\mathrm{p}=0.005)$, insulin $(\mathrm{p}=0.019)$ and antianginals $(p=0.025)$. In the univariate analysis, use of antihypertensives or statins during the IBP did not have any role on NPADM. The results of the Cox proportional hazards analysis (figure 6) showed that NPADM incidence was significantly associated with age, sex and the following prescribed medications during the IBP: statins (HR: 0.82, $95 \%$ CI 0.73 to $0.92 ; \mathrm{p}=0.001$ ), antihypertensives (HR: 

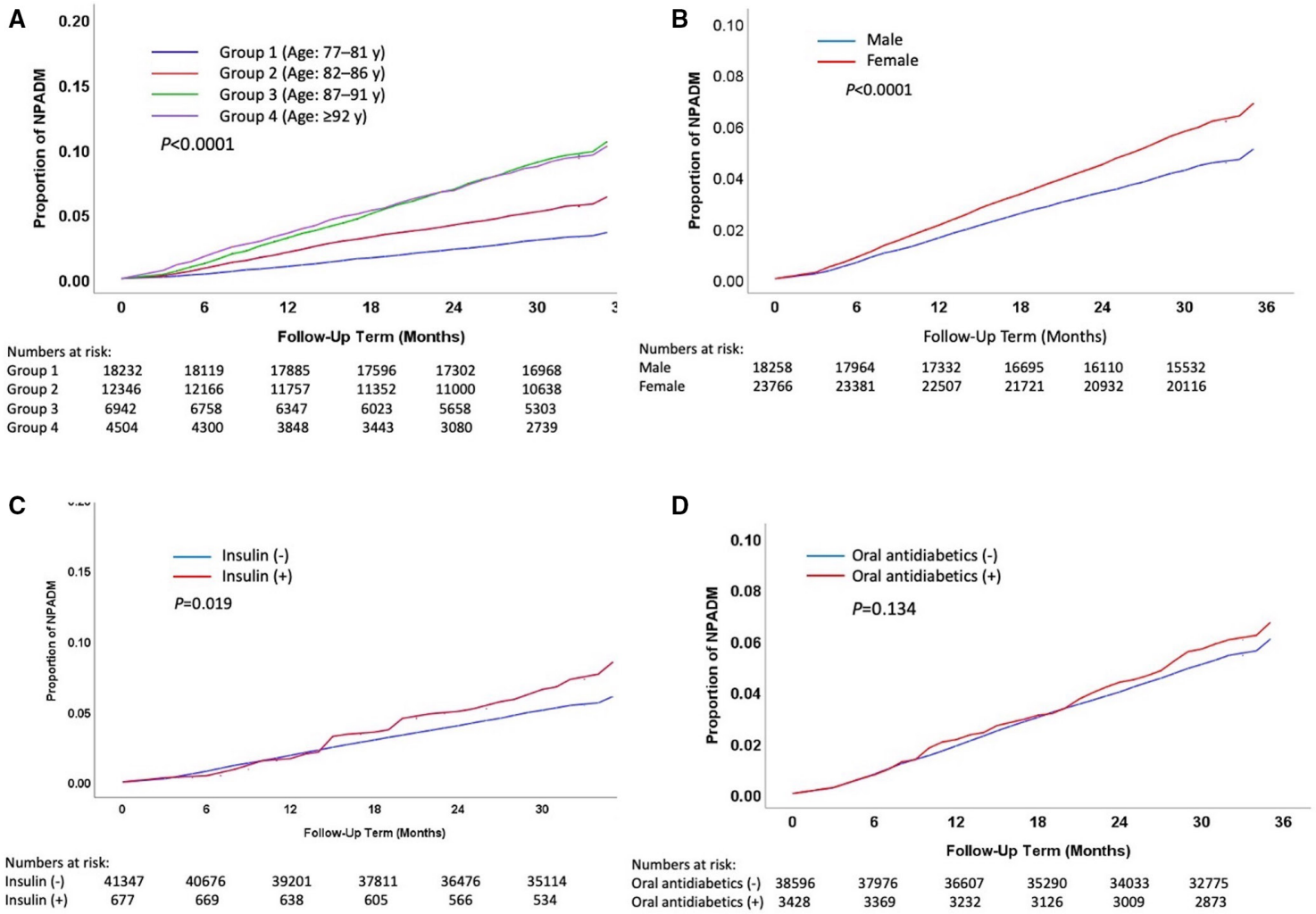

Figure 2 Kaplan-Meier curves showing the cumulative incidence of NPADM stratified by subject characteristics and use of antidiabetics. Cumulative incidence of NPADM stratified by (A) age group, (B) sex, (C) use of insulin and (D) use of oral antidiabetics during the initial background period (from 1 April to 30 June 2012). NPADM, new prescription of antidementia medication.

$0.80,95 \%$ CI 0.73 to 0.88 ; $\mathrm{p}<0.0001)$, non-steroidal bronchodilators (HR: $0.72,95 \%$ CI 0.58 to 0.88 ; $\mathrm{p}=0.002$ ), antidepressants (HR: $1.79,95 \%$ CI 1.47 to $2.18 ; \mathrm{p}<0.0001$ ), poststroke medications (HR: $1.45,95 \%$ CI 1.16 to 1.82; $\mathrm{p}=0.002$ ), insulin (HR: $1.34,95 \%$ CI 1.01 to 1.78 ; $\mathrm{p}=0.046$ ) and antineoplastics (HR: $1.12,95 \%$ CI 1.01 to $1.24 ; \mathrm{p}=0.035$ ).

The results of an ad hoc analysis including an interaction term (age*sex) in the multivariable analysis are available in online supplemental material 2. The age and sex interaction did not reach significant level.

\section{DISCUSSION}

In this retrospective cohort study of older adults residing in a Japanese city, we examined the associations of use of prescription medications for existing chronic conditions (indicating the pharmaceutical management of dementia risk factors) with NPADM (indicating progression to clinical dementia). The NPADM incidence was $1.9 \%$ at 12 months after study initiation, and this incidence in subjects aged 87 years or older was more than three times higher than that of younger subjects aged 77-81 years. NPADM incidence was significantly higher in women and subjects who were treated with insulin, antidepressants, antineoplastics and poststroke medications during the IBP. In contrast, NPADM incidence was significantly lower in subjects who were treated with statins, antihypertensives and non-steroidal bronchodilators during IBP. While use of steroids ${ }^{6}$ benzodiazepines, ${ }^{7}$ anticoagulants ${ }^{8}$ and nonsteroidal anti-inflammatory drugs ${ }^{9}$ is reportedly associated with the development of Alzheimer's disease, our analysis did not detect any significant associations with NPADM.

The results of this study should be cautiously interpreted with the understanding that the effects of the target medications on NPADM cannot be separated from those of the underlying conditions. Previous studies have asserted that controlling cardiovascular risk factors can mitigate the onset and progression of Alzheimer's disease. ${ }^{10} 11$ Based on those findings, we included antianginals, antiplatelets, anticoagulants, antihypertensives and statins in our analysis. However, the multivariate analysis showed that only statins, antihypertensives and non-stroiedal bronchodilators were significantly associated with lower incidence of NPADM (risk reductions of $18 \%$ for statins and $20 \%$ for antihypertensives). In contrast to our findings, two prospective randomised controlled trials failed to demonstrate the benefits of statins in the prevention of dementia, ${ }^{12}{ }^{13}$ although beneficial effects were indicated by a cohort study. ${ }^{14}$ Our analysis differed from the two trials in that our subjects were already taking statins during the IBP, whereas statins were prescribed to the trial participants only after randomisation. ${ }^{12} 13$ Next, our analysis found that use of non-steroidal bronchodilators 
Table 1 Sex and prescribed medications during the IBP in all subjects and according to age group

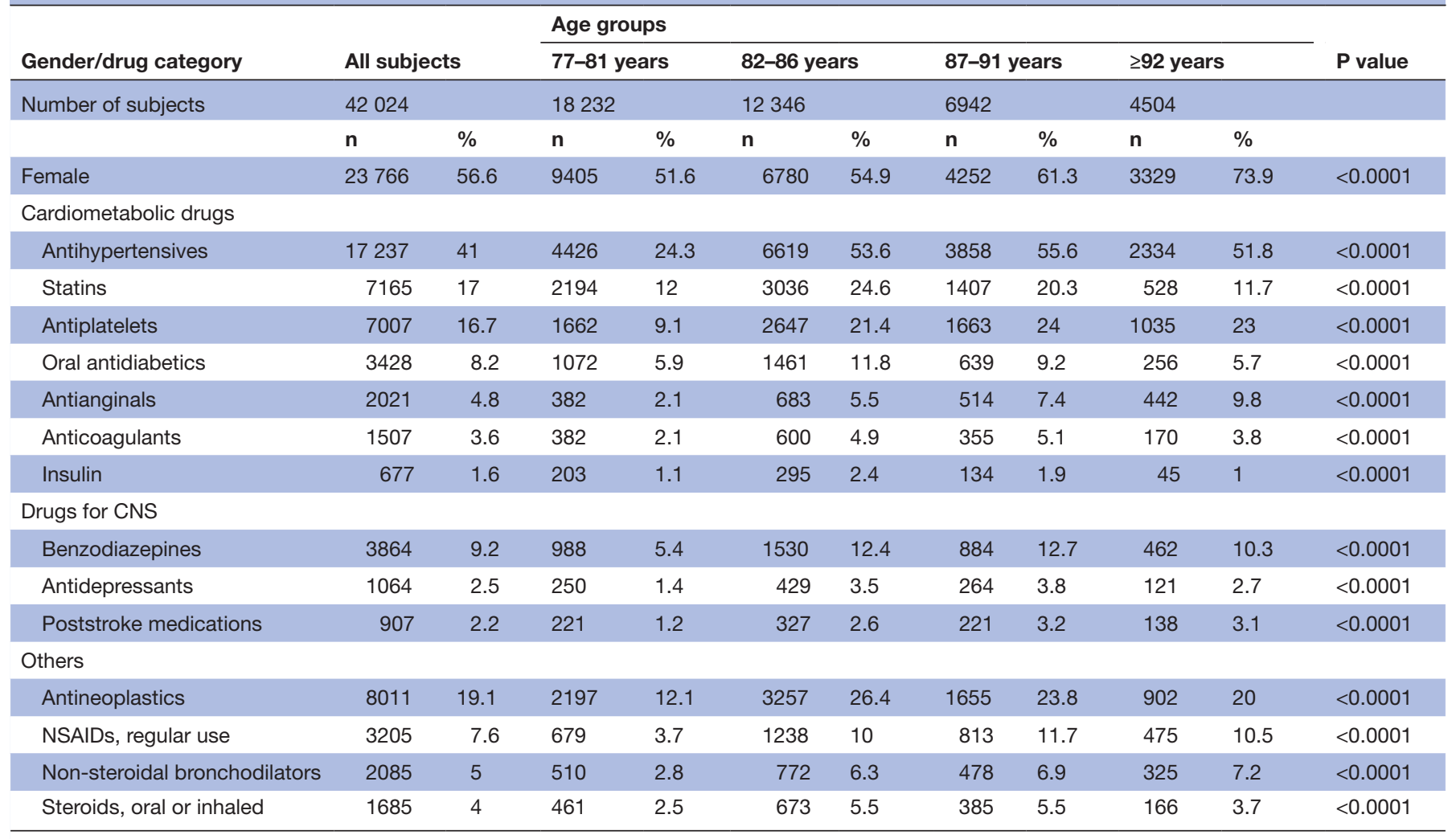

CNS, central nervous system; IBP, initial background period (from 1 April to 30 June 2012); NSAID, non-steroidal anti-inflammatory drug.

was associated with a reduced incidence of NPADM. Many researchers suspect that use of medications which have anticholinergic property is associated with increased risk of dementia. In the study, the use of non-steroidal bronchodilators, which have $\beta$-adrenergic stimulant as well as anticholinergic properties, did not increase NPADM, but reduced it. Meanwhile, Japanese package inserts for antidementia medications note that it must be cautiously prescribed to patients who are taking bronchodilators for chronic obstructive pulmonary disease or asthma. ${ }^{15} \mathrm{We}$ posit that the reduced incidence of NPADM in subjects being treated with non-steroidal bronchodilators may also be indicative of physicians' caution in prescribing antidementia medications.

Diabetes is a well-established risk factor for dementia, including Alzheimer's disease. ${ }^{12}$ Insulin is generally used for patients who are unable to manage their blood glucose levels through the use of oral antidiabetics alone. In addition to its hypoglycaemic properties, oral antidiabetics (sulfonylureas and metformin) have been reported to substantially reduce the risk of dementia. ${ }^{16}$ It is therefore possible that subjects who require insulin have a higher risk of dementia than those taking oral antidiabetics as the latter could mitigate the risk of diabetes-associated dementia. History of stroke is another known risk factor for cognitive decline and its progression to dementia. ${ }^{17}$ In Japan, medications to stimulate brain metabolism or increase cerebrovascular blood flow are prescribed to patients with a history of stroke. Our study subjects who were already taking these poststroke medications had an elevated risk of NPADM. Antidepressants and benzodiazepines are widely used when the diagnosis of Alzheimer's disease was made. ${ }^{18}$ The incidence of antidepressant use was higher in persons with Alzheimer's disease than in persons without. ${ }^{19}$ In the present study, antidepressant use was associated with increased incidence of NPADM, while benzodiazepine was not. It is currently under discussion as to whether depression is a risk factor or prodrome of dementia. ${ }^{2}$ In addition, the current data set included $32 \%$ of tricyclic antidepressants, which have a strong anticholinergic property and might have affected the increased incidence of NPADM (online supplemental material $3)$. Further study is required on this topic. In summary, although the present cohort study only provides the association between coprescribed medication and NPADM as an observational study, the results in a real-world setting of a Japanese city do not contradict the recommendations of the WHO. ${ }^{1}$

Despite being widely prescribed, the currently available antidementia medications for Alzheimer's disease and Lewy body disease only provide symptomatic treatment without modifying the underlying neurodegenerative process. $^{2}$ As there are no effective curative treatments at present, the WHO has recommended multidomain strategies that incorporate both lifestyle modifications and pharmaceutical management to reduce new cases of dementia and its progression. ${ }^{1}$ Lifestyle management recommended to reduce the risk of dementia was applied 
A

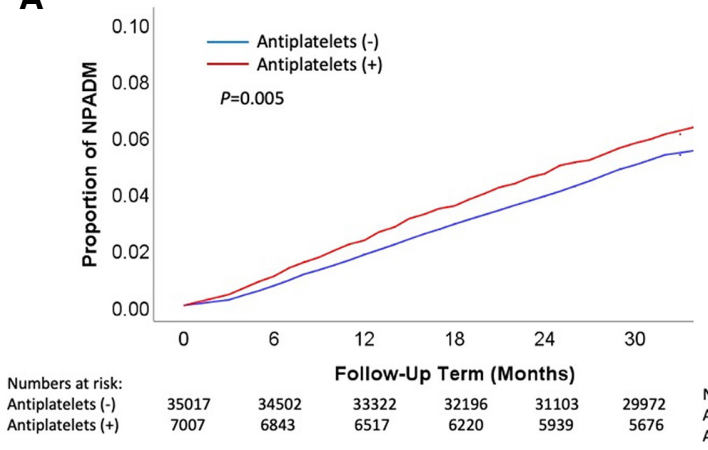

B

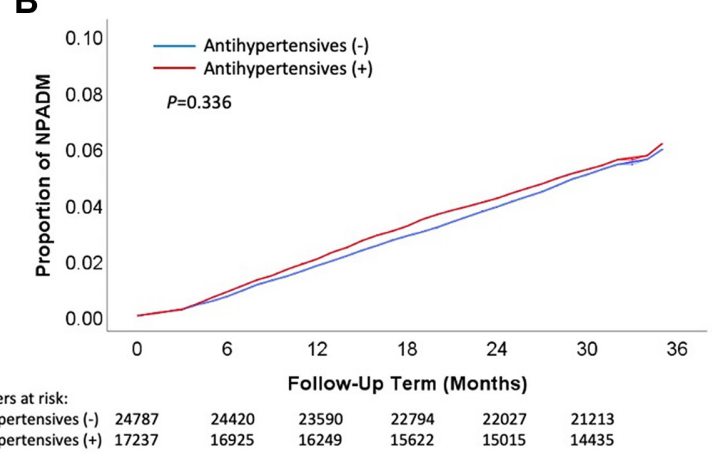

\section{C}

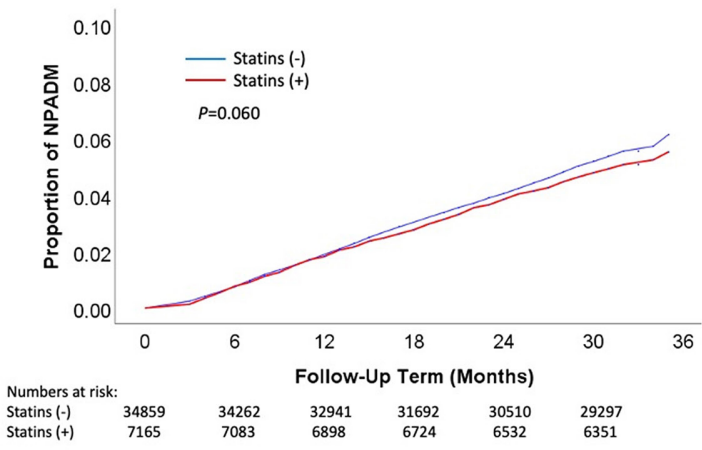

D

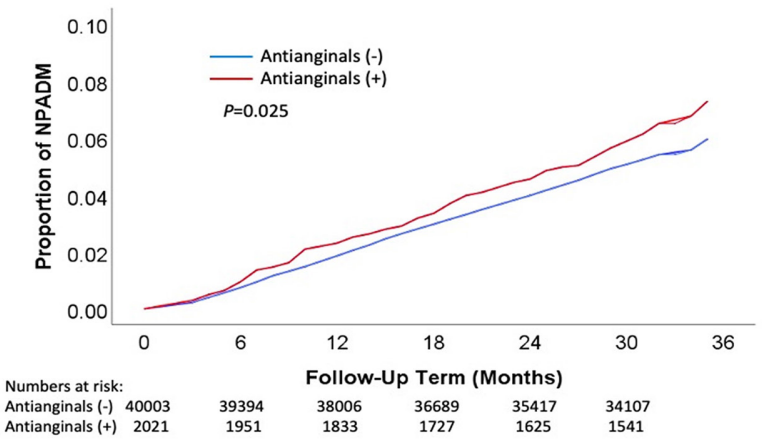

Figure 3 Kaplan-Meier curves showing the cumulative incidence of NPADM stratified by use of blood and cardiovascular system medications. Cumulative incidence of NPADM stratified by use of (A) antiplatelets, (B) antihypertensives, (C) statins and (D) antianginals during the initial background period (from 1 April to 30 June 2012). NPADM, new prescription of antidementia medication.

for, obesity and appropriate intake of alcohol in mid-life, smoking cessation, maintaining physical activity, avoiding social isolation and air pollution. To treat depression in later life and to use hearing aid for hearing loss are also recommended. ${ }^{12}$ Intervention of these modifiable factors can potentially reduce the risk of dementia by $40 \%{ }^{2}$ Several prospective randomised trials have assessed the effectiveness of such interventions to prevent cognitive decline but have produced conflicting results; the Finnish Geriatric Intervention Study to Prevent Cognitive Impairment and Disability (FINGER) multidomain lifestyle intervention (FINGER) trial reported a reduction in risk of dementia, ${ }^{20}$ but two other studies found no significant effects on dementia incidence or cognitive decline. ${ }^{21}{ }^{22}$

Our study design is characterised by several features. First, under Japan's universal healthcare system, all Kashiwa City residents aged 75 years or older are, without exception, covered by the LLMCS until death, unless they move out of the city or are admitted to a nursing facility. The LLMCS is managed by a single payer (ie, the Kashiwa City government), which integrates claims in different medical institutions. Therefore, a strength of this study is the use of an exhaustive survey of insurance claims data. Second, it is difficult to obtain accurate diagnoses of Alzheimer's disease or Lewy body disease without access to the complete set of clinical evaluations by physicians, laboratory test results, cognitive test results and neurological images. It is also essentially impossible to identify the point at which mild cognitive impairment progresses to clinical dementia. Accordingly, we employed NPADM as a proxy to identify this progression. Third, we focused on older adults (aged $\geq 77$ years), who often have multiple existing conditions that are treated with various medications. We examined 14 categories of medications for commonly observed conditions in the target population. In order to ensure the accuracy of identifying patients with specific conditions in the target population, we only used prescription data that are directly linked with reimbursements. Diagnostic codes were not used to identify patients with specific conditions because providers may assign these codes for the sole purpose of reimbursement. We believe this approach effectively uses the accurate and reliable information available in Japanese claims records. Efforts to integrate claims records and clinical information from electronic health records are needed to support more accurate population studies that would shed light on the effectiveness and safety profile of various therapies. The Pharmaceuticals and Medical Devices Agency of Japan established the Medical Information Database Network Project to integrate these data sets in selected medical institutions and is being used for postmarketing surveillance of marketing authorisation holder products. ${ }^{23}$

Several higher-income countries (including the USA, the UK, Sweden, the Netherlands and Canada) have recently reported reductions in the incidence of 

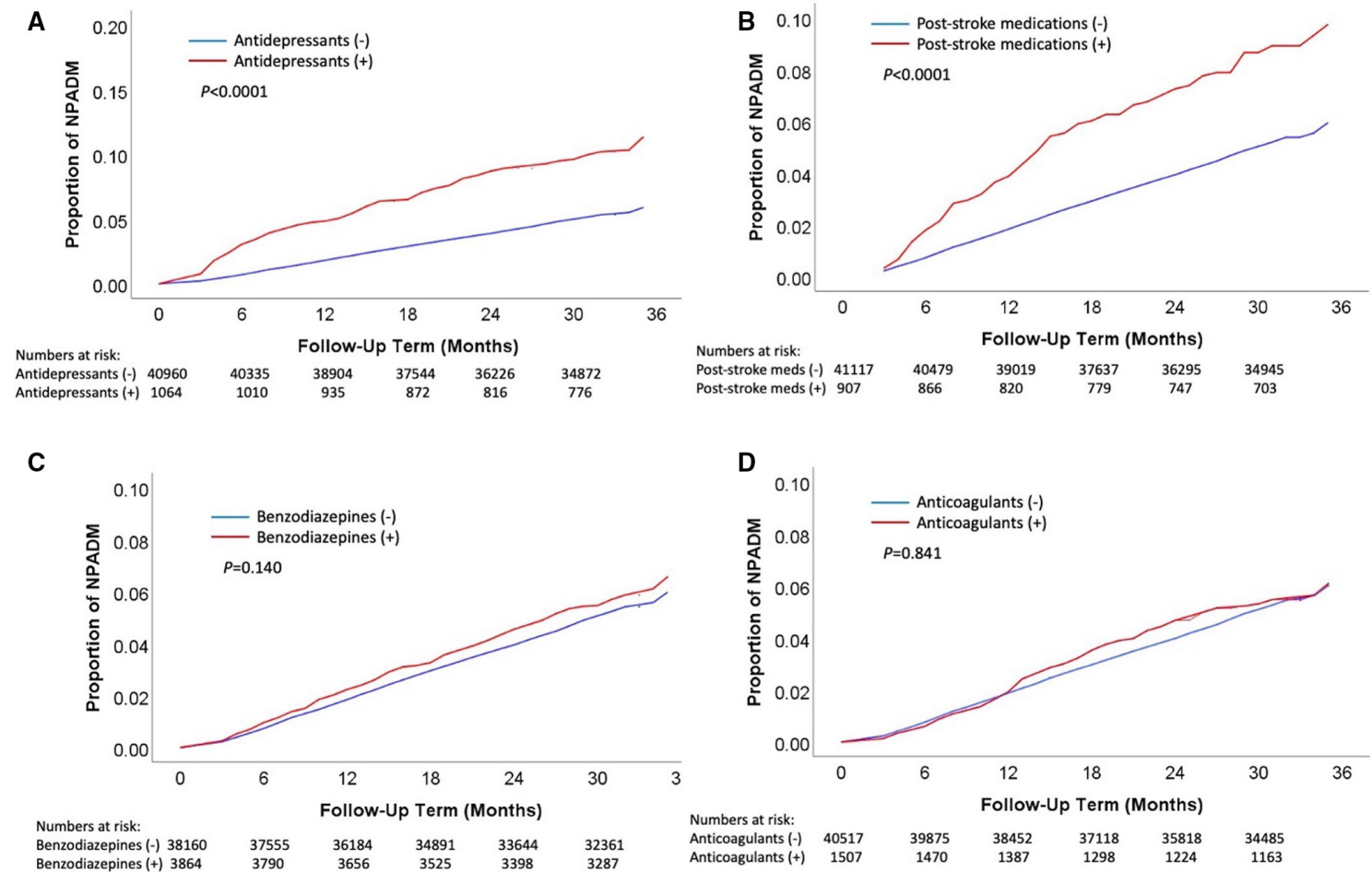

Figure 4 Kaplan-Meier curves showing the cumulative incidence of NPADM stratified by use of brain/nervous system and dyslipidaemia medications. Cumulative incidence of NPADM stratified by use of (A) antidepressants, (B) poststroke medications, (C) benzodiazepines and (D) anticoagulants during the initial background period (from 1 April to 30 June 2012). NPADM, new prescription of antidementia medication.
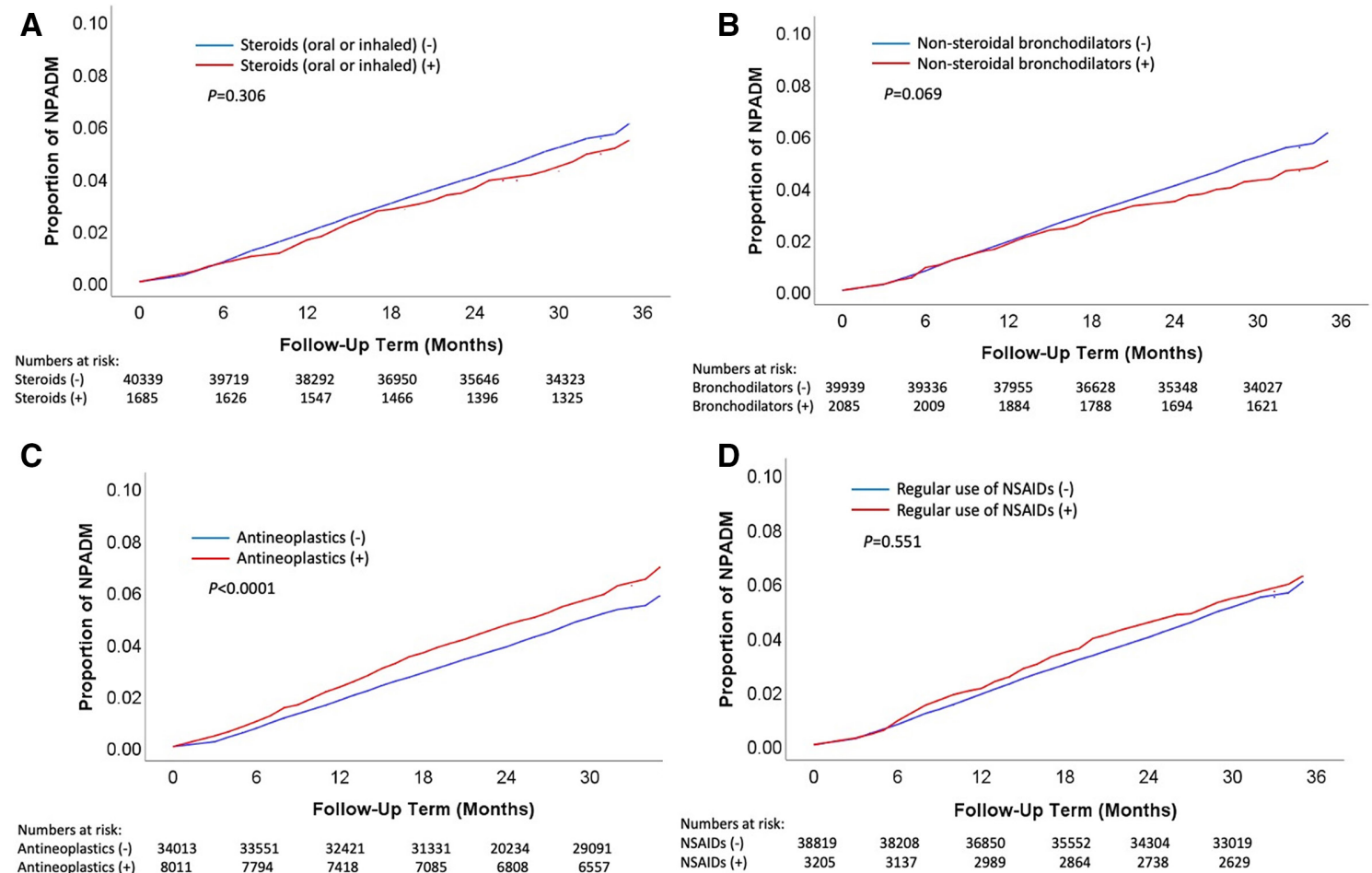

Figure 5 Kaplan-Meier curves showing the cumulative incidence of NPADM stratified by use of steroids, anti-inflammatory drugs, antineoplastics and bronchodilators. Cumulative incidence of NPADM stratified by use of (A) steroids (oral or inhaled), (B) non-steroidal bronchodilators, (C) antineoplastics and (D) NSAIDs during the initial background period (from 1 April to 30 June 2012). NPADM, new prescription of antidementia medication; NSAID, non-steroidal anti-inflammatory drug. 


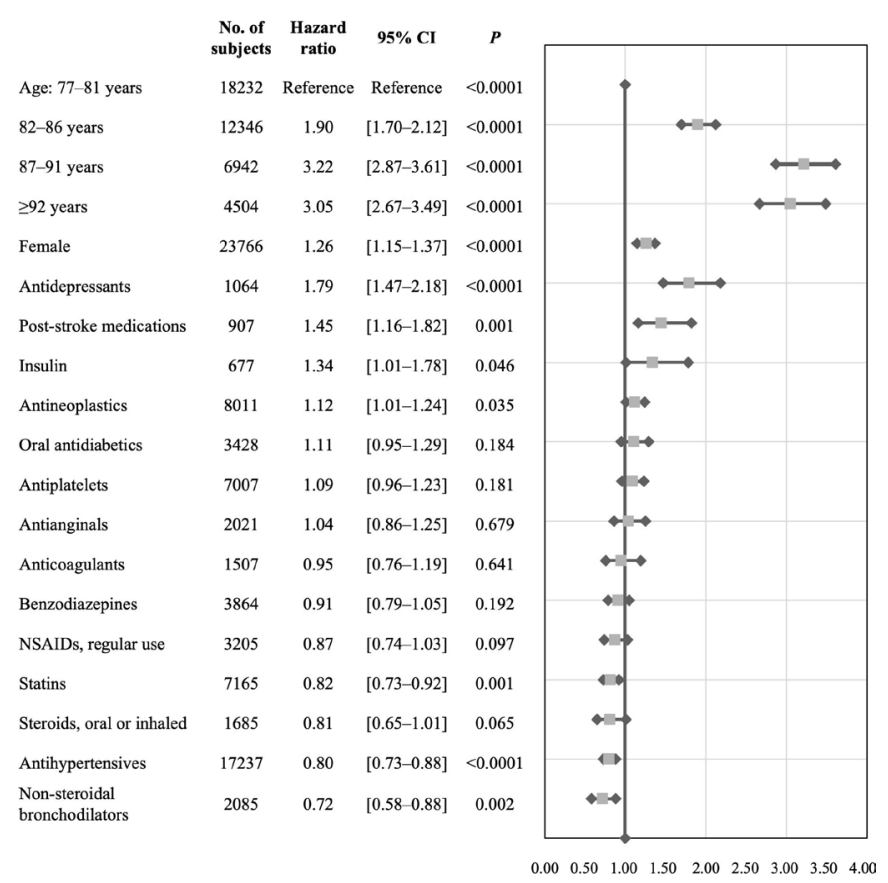

Figure 6 Results of the Cox proportional hazards analysis of incidence of new prescription of antidementia medication. NSAID, non-steroidal anti-inflammatory drug.

dementia, ${ }^{2}$ but its prevalence is increasing in Japan. ${ }^{24}{ }^{25}$ The Hisayama Study is a population-based study of a rural Japanese town (mean age: 76 years) that examines the risk factors for various diseases, including dementia. ${ }^{26}$ The analyses of this Japanese legacy database reported only the prevalence of Alzheimer's disease (12.3\%). The Japan Prospective Studies Collaboration for Aging and Dementia, consisting of more than 10000 subjects aged more than 65 years, is ongoing and is designed to assess the incidence of dementia and potentially modify its disease process. ${ }^{27}$

\section{Limitations}

The study has several limitations. First, administrative claims collected in real-world clinical practice are not designed for research purpose. As the study design is a retrospective cohort study, the cause-effect relationship was not determined. Therefore, the results only described the association between coprescribed medications and onset of clinical dementia indicated by NPADM. Second, the effects of each medication could not be distinguished from the underlying pathophysiological condition for which it was prescribed. For example, NPADM in patients taking antidiabetics may be affected by the medication, diabetes or a combination thereof. In addition, the control of blood glucose levels is likely to be an important aspect of reducing risk of dementia, but this information was not available in our data set. Third, although we assume that NPADM captured the progression from asymptomatic into symptomatic dementia, it is entirely dependent on the decisions of individual physicians, such as neurologists, psychiatrists and primary care physicians. Various guidelines include recommendations on the prescription and discontinuation of cholinesterase inhibitors and N-methyl-d-aspartate receptor antagonists. ${ }^{25}{ }^{28}$ However, the decision-making process varied among subspecialties and physicians. Fourth, anticholinergic medications are associated with risk of dementia. ${ }^{29}$ However, it is not yet well understood what level of anticholinergic property and what class of medication have increased risk of dementia. We included antidepressants and bronchodilators with anticholinergic properties, while other classes of drugs such as those for Parkinson's disease and peptic ulcer were not included in the study. Estimating the level of anticholinergic in each drug and evaluating the effect on NPADM are out of scope of the present study. Fifth, although antidementia medications are only approved for Alzheimer's disease and Lewy body disease following Japanese regulatory approval, we cannot exclude the possibility that physicians may prescribe these medications to treat vascular dementia. As the study population consists of very old adults ( $\geq 77$ years old), it is supposed that a substantial proportion of study subjects have combined pathology of vascular dementia as well as Alzheimer disease and Lewy body disease. Although poststroke medications prescribed for patients with a history of stroke reflected the vascular component of dementia, no obvious history of stroke such as multiple lacunar infarcts may be overlooked in the study. Finally, $2.1 \%$ of study subjects $(n=879)$ were censored because they were admitted into a nursing facility during the study period because they were covered by the long-term care insurance system for disabled subjects after admission. Most of them were admitted into nursing homes due to impairment in physical function, while some might have concomitant cognitive decline. As the incidence of NPADM in older adults who reside in nursing facility is assumed to be higher than in community-dwelling subjects, the effect of medications assessed in the study might have been underestimated.

\section{Implications}

We examined the associations of the use of prescription medications for existing chronic diseases with NPADM in the complete enumeration cohort study of insurance claims data from older adults. Although the study was only a retrospective cohort study, an informative association between several coprescribed medications for chronic comorbid conditions and NPADM in the very old adults was identified. The findings of this populationbased natural experiment were generally consistent with the WHO recommendations for the control of dementia risk factors. We believe that our findings would be helpful to better understand the current clinical practice for dementia in the real-world setting and potentially contribute to healthcare policymaking.

\section{Author affiliations}

${ }^{1}$ Human Care Research Team, Tokyo Metropolitan Institute of Gerontology, Tokyo, Japan

${ }^{2}$ Medical Technology Innovation Centre, Juntnndo University, Tokyo, Japan

${ }^{3}$ Pharmaceuticals and Medical Devices Agency, Tokyo, Japan 
${ }^{4}$ Department of Geriatric Medicine, Clinic lan South Center, Yokohama, Kanagawa, Japan

${ }^{5}$ Department of Geriatric Medicine, Graduate School of Medicine, The University of Tokyo, Tokyo, Japan

${ }^{6}$ Health Services Research, University of Tsukuba, Tsukuba, Japan

${ }^{7}$ Institute of Gerontology, University of Tokyo, Tokyo, Japan

${ }^{8}$ Institute for Future Initiatives, The University of Tokyo, Tokyo, Japan

Acknowledgements We thank Dr Syunei Kyo for his support to our research team. The views expressed in this article are those of the authors and do not necessarily reflect the official views of the PMDA.

Contributors NH: conception and design of the work, data acquisition, analysis and interpretation of data, medical writing, editing the manuscript. SM: conception and design of the work, data acquisition, analysis and interpretation of data, critical revision, editing the manuscript. Tl: conception and design of the work, acquisition, analysis or interpretation of data for the work, editing the manuscript, critical revision, final approval. TN, MA, NT, SY, KI: analysis or interpretation of data for the work, editing the manuscript, critical revision.

Funding The cost of conducting the study was supported by KAKENHI Grant-in-Aid for Scientific Research (19K22790 and 20H03923) from Japan Society for the Promotion of Science

Competing interests MA has received grants from Astellas Pharma, Bayer HealthCare, Boehringer Ingelheim, Chugai Pharmaceutical, Daiichi Sankyo, Eli Lilly Japan, Eisai, Kracie Pharma, Mitsubishi Tanabe Pharma, MSD, Novartis Japan, Ono Pharmaceutical, Sanofi, Teijin Pharma and Tsumura, and personal fees from Daiichi Sankyo, Mitsubishi Tanabe Pharma, MSD, Takeda Pharmaceutical and Sumitomo Dainippon Pharma, outside the submitted work. The remaining authors have nothing to disclose.

Patient consent for publication Not required.

Ethics approval The study obtained ethics approval from the ethical committee of Kashiwa City government. As the study used anonymised insurance claims data, opt-in informed consent was waived. The study was also approved by the institutional ethics committees of the Tokyo Metropolitan Institute of Gerontology (approval no. 28: 16 0ctober 2015), where this study was performed.

Provenance and peer review Not commissioned; externally peer reviewed.

Data availability statement Data are available upon reasonable request. All data underlying this study are owned by the Kashiwa City government. The study was conducted in accordance with a contract between the Kashiwa City government and the Tokyo Metropolitan Geriatric Medical Center and Institute of Gerontology. The contract stipulates that the authors are prohibited from using the data for any purpose other than this study or providing the data to anyone other than the approved research group members without the express permission of the Kashiwa City government. Researchers interested in the data should contact the project leader $(\mathrm{TI})$.

Supplemental material This content has been supplied by the author(s). It has not been vetted by BMJ Publishing Group Limited (BMJ) and may not have been peer-reviewed. Any opinions or recommendations discussed are solely those of the author(s) and are not endorsed by BMJ. BMJ disclaims all liability and responsibility arising from any reliance placed on the content. Where the content includes any translated material, BMJ does not warrant the accuracy and reliability of the translations (including but not limited to local regulations, clinical guidelines, terminology, drug names and drug dosages), and is not responsible for any error and/or omissions arising from translation and adaptation or otherwise.

Open access This is an open access article distributed in accordance with the Creative Commons Attribution Non Commercial (CC BY-NC 4.0) license, which permits others to distribute, remix, adapt, build upon this work non-commercially, and license their derivative works on different terms, provided the original work is properly cited, appropriate credit is given, any changes made indicated, and the use is non-commercial. See: http://creativecommons.org/licenses/by-nc/4.0/.

ORCID iD

Nobuhiro Handa http://orcid.org/0000-0003-2838-6462

\section{REFERENCES}

1 Risk reduction of cognitive decline and dementia: who guidelines, 2019. World Health organization. Available: https://apps.who.int/ iris/bitstream/handle/10665/312180/9789241550543-eng.pdf?ua=1 [Accessed 20 Feb 2021].

2 Livingston G, Huntley J, Sommerlad A, et al. Dementia prevention, intervention, and care: 2020 report of the Lancet Commission. Lancet 2020;396:413-46.

3 Joe E, Ringman JM. Cognitive symptoms of Alzheimer's disease: clinical management and prevention. BMJ 2019;367:16217.

4 Outline of health care insurance system, 2009. Available: https:// www.mhlw.go.jp/english/wp/wp-hw3/dl/2-001.pdf [Accessed 20 Feb 2021]

5 Mitsutake S, Ishizaki T, Teramoto C, et al. Patterns of co-occurrence of chronic disease among older adults in Tokyo, Japan. Prev Chronic Dis 2019;16:E11.

6 Nerius M, Haenisch B, Gomm W, et al. Glucocorticoid therapy is associated with a lower risk of dementia. J Alzheimers Dis 2020;73:175-83.

7 Ding M, Fratiglioni L, Johnell K, et al. Atrial fibrillation, antithrombotic treatment, and cognitive aging: a population-based study. Neurology 2018;91:e1732-40.

8 Zhong G, Wang Y, Zhang Y, et al. Association between benzodiazepine use and dementia: a meta-analysis. PLoS One 2015;10:e0127836. eCollection.

9 Wang J, Tan L, Wang H-F, et al. Anti-inflammatory drugs and risk of Alzheimer's disease: an updated systematic review and metaanalysis. J Alzheimers Dis 2015;44:385-96.

10 Samieri C, Perier M-C, Gaye B, et al. Association of cardiovascular health level in older age with cognitive decline and incident dementia. JAMA 2018;320:657-64.

11 Sabia S, Fayosse A, Dumurgier J, et al. Association of ideal cardiovascular health at age 50 with incidence of dementia: 25 year follow-up of Whitehall II cohort study. BMJ 2019;366:I4414.

12 Heart Protection Study Collaborative Group. MRC/BHF heart protection study of cholesterol lowering with simvastatin in 20,536 high-risk individuals: a randomised placebo-controlled trial. Lancet 2002;360:7-22.

13 Shepherd J, Blauw GJ, Murphy MB, et al. Pravastatin in elderly individuals at risk of vascular disease (prosper): a randomised controlled trial. Lancet 2002;360:1623-30.

14 Zhang X, Wen J, Zhang Z. Statins use and risk of dementia: a doseresponse meta analysis. Medicine 2018;97:e11304.

15 Package inserts: Aricept (Japanese), 2017. Available: https://www. info.pmda.go.jp/go/pack/1190012C1020_1_27/ [Accessed 20 Feb 2021].

16 Hsu C-C, Wahlqvist ML, Lee M-S, et al. Incidence of dementia is increased in type 2 diabetes and reduced by the use of sulfonylureas and metformin. J Alzheimers Dis 2011;24:485-93.

17 Zhou J, Yu J-T, Wang H-F, et al. Association between stroke and Alzheimer's disease: systematic review and meta-analysis. $J$ Alzheimers Dis 2015;43:479-89.

18 Tolppanen A-M, Taipale H, Koponen M, et al. Cohort profile: the finnish medication and Alzheimer's disease (MEDALZ) study. BMJ Open 2016;6:e012100.

19 Puranen A, Taipale H, Koponen M, et al. Incidence of antidepressant use in community-dwelling persons with and without Alzheimer's disease: 13-year follow-up. Int J Geriatr Psychiatry 2017;32:94-101.

20 Ngandu T, Lehtisalo J, Solomon A, et al. A 2 year multidomain intervention of diet, exercise, cognitive training, and vascular risk monitoring versus control to prevent cognitive decline in at-risk elderly people (FINGER): a randomised controlled trial. Lancet 2015;385:2255-63.

21 Moll van Charante EP, Richard E, Eurelings LS, et al. Effectiveness of a 6-year multidomain vascular care intervention to prevent dementia (preDIVA): a cluster-randomised controlled trial. Lancet 2016;388:797-805

22 Andrieu S, Guyonnet S, Coley N, et al. Effect of long-term omega 3 polyunsaturated fatty acid supplementation with or without multidomain intervention on cognitive function in elderly adults with memory complaints (MAPT): a randomised, placebo-controlled trial. Lancet Neurol 2017;16:377-89.

23 Yamaguchi M, Inomata S, Harada S, et al. Establishment of the MID-NET ${ }^{\circledR}$ medical information database network as a reliable and valuable database for drug safety assessments in Japan. Pharmacoepidemiol Drug Saf 2019;28:1395-404.

24 Okamura H, Ishii S, Ishii T, et al. Prevalence of dementia in Japan: a systematic review. Dement Geriatr Cogn Disord 2013;36:111-8.

25 Dementia: assessment, management and support for people living with dementia and their carers. NICE guideline. Available: https:// www.nice.org.uk/guidance/ng97 [Accessed 20 Feb 2021].

26 Ninomiya T. Japanese legacy cohort studies: the Hisayama study. J Epidemiol 2018;28:444-51. 
27 Japan prospective studies collaboration for ageing and dementia (JPSC-AD) (Japanese). Available: https://www.eph.med.kyushu-u.ac. jp/jpsc/en/ [Accessed 20 Feb 2021].

28 Petersen RC, Lopez O, Armstrong MJ, et al. Practice guideline update summary: mild cognitive impairment: report of the
Guideline development, Dissemination, and Implementation Subcommittee of the American Academy of Neurology. Neurology 2018;90:126-35.

29 Richardson K, Fox C, Maidment I, et al. Anticholinergic drugs and risk of dementia: case-control study. BMJ 2018;29:k1315. 